\title{
Development of Partial Tubular Flat Knitting Fabric Composite Preform
}

\author{
Wei Qing JIANG ${ }^{1}$ Chun Xia CHEN ${ }^{2}$ \\ ${ }^{1}$ Yan cheng institute of industry College, Yan cheng, Jiang su 224005 \\ ${ }^{2}$ Excellent Scientific and Technological Innovation Team of Jiangsu Province 2015
}

\begin{abstract}
After building some structures of partial tubular flat knitting fabric composite preform, the influencing factor on tubular section was analyzed and the fabric was knitted selectively. The partial tubular flat knitting fabric composite preform were Knitted by changing different yarn, row number and two-sided partial tubular flat knitting fabric. Multilayer sheet would be got after hot pressing and it has big market prospects and good application value.

Key words: partial tubular; flat knitting fabric; composite preform; structure design; development

Top-notch Academic Programs Project of Jiangsu Higher Education Institutions 2015
\end{abstract}

\section{Development of composite preform}

In recent years, the thermoplastic composites material which is used textile preform as reinforcement develop rapidly, for the industrial use of textiles, the proportion is also increasing. Braided、woven and knitted fabric construction are often used as textile preform. among the Knitted fabric preforms ,warp knitted fabric preform's development is much maturer, because the weft knitted fabric preform has good extension flexibility and high production efficiency, so it has broad production prospects.

At present, the research on the whole tubular structure of flat knitting fabric has been made at home and abroad. This knitting method achieve braiding cylindric structure on the knitting machine. The fabric is a whole tube. But the report of the structure of partial tubular flat knitting fabric as well as the fabric is few. For this paper the partial tubular flat knitting fabric composite preform structure is designed and trial-weaving. The purpose is to lay the foundation for the 3D knitting fabric production and industrialization promotion of the composite materials.

\section{Structure design of partial tubular flat knitting fabric composite preform}

The structure of partial tubular flat knitting fabric was designed, as shown in figure 1. 


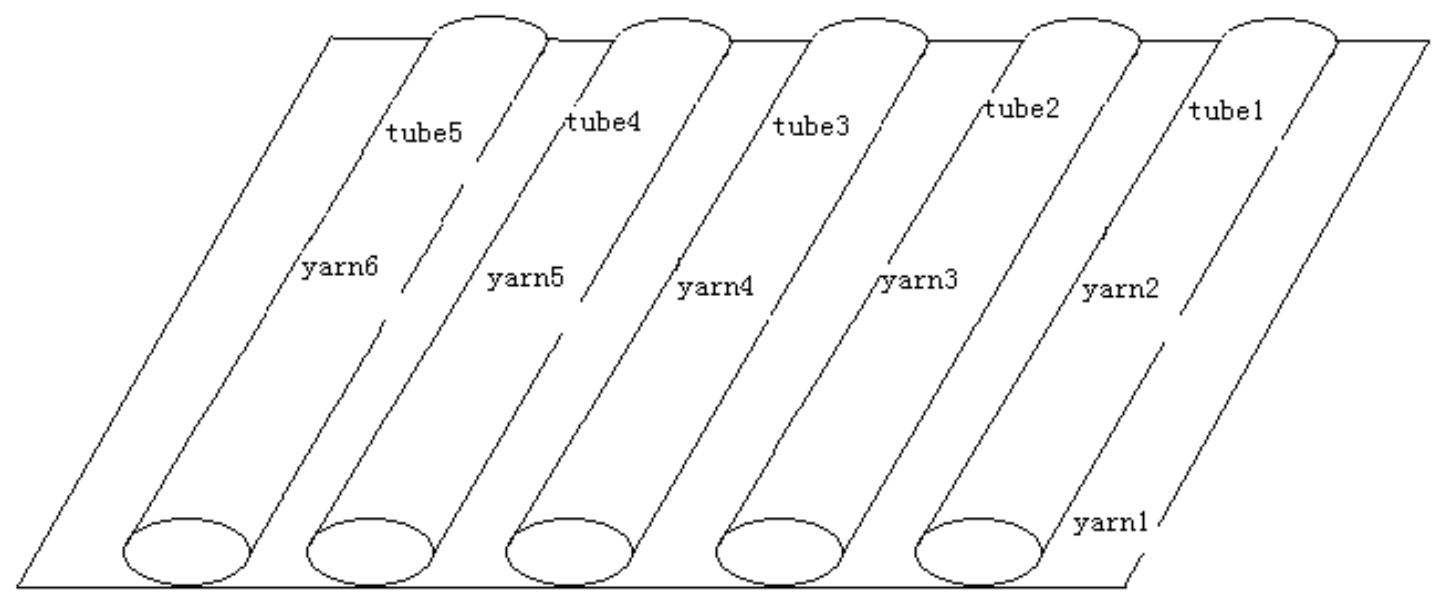

figure 1 Structure of partial tubular flat knitting fabric composite preform

In Figure 1, the yarn 1 is woven in the plane part of partial tubular flat knitting fabric composite preform. partial tubular structure 1-5 Knitted with yarn 2-6, The appearance presented by the tubular part mainly affected by yarn, fabric weave, row number etc.

For the Structure design of the partial tubular flat knitting fabric composite preform, the following changes can be choosed, Yarn 1-6 can choose the same or different colors and raw materials for weaving, every parts of tube 1-5 different can use different fabric structure.

\section{The machine design process of partial tubular flat knitting fabric composite preform}

partial tubular flat knitting fabric composite preform were knitted on a flat knitting machine. Taking organization cycle of 160 row circular as example, the knitting diagram is shown in Figure 2. When specific weaving, Organization cycle course number、 yarn number that participate in knitting etc.can be designed according to the specific needs.

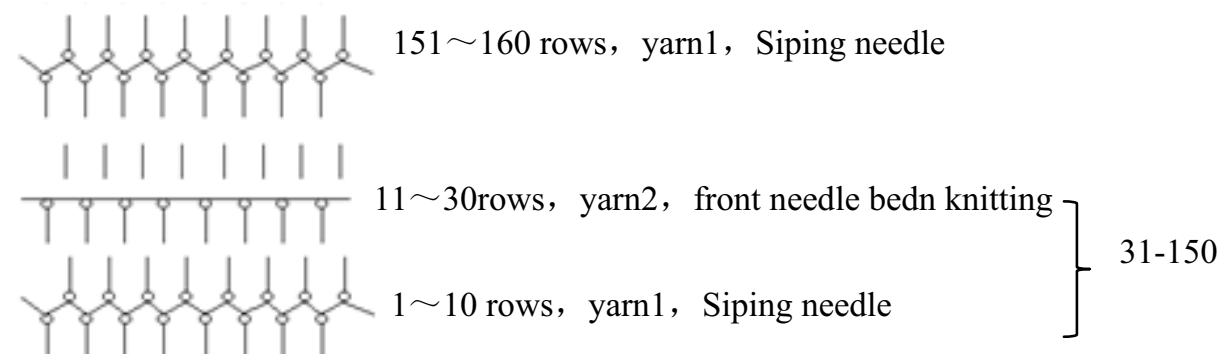

rows, repeats the process

Figure 2 knitting diagram of partial tubular flat knitting fabric composite preform partial tubular flat knitting fabric composite preform knitting process is as follows

\section{1 knitting of one pipe structure} for the 1-50 longitudinal yarn, take it turns to knit10 rows by using the yarn 1 before and after needle bed, Siping needle。 when knitting the 11-30 rows, close the knitting triangle of the back needle bed. change the Yarn nozzle 2, Knitting with yarn 2, Pay attention to give the tubular part pressure during the knitting process. In order to avoid the fabric floating on the fell affect knitting.the 30th rows, After opening the needle bed knitting triangle. Woven to form a partial tubular structure.

\section{2multiple partial tubulars knitting}

From the thirty-first row, according to figure 2 alternately repeating one pipe structure knitting, Successive formation of multiple partial tubulars structures, for the example of this paper, yarn2-yarn6 selected dfferent colors of yarn respectively, we can choose different colors, different raw materials, different thickness of the 
yarn according to the need of the Actual design 。

\section{Trial knitting of partial tubular flat knitting fabric composite preform}

\section{1 machine conditions}

the design and trial knitting of the partial tubular flat knitting fabric composite preform can be knitting on hand-operated flat knitting machines or computerized flat knitting machines, The machine conditions are as follows.

\subsection{1 hand-operated flat knitting machines} Machines: Nan tong sansi Technology, ongxing brand hand-operated flat knitting machines Yarns: 28s/2、White, green, pink, red, blue, orange, mulberry /PP blended yarn

Machine No.: E9。

\subsection{2computerized flat knitting machines} Machines: Jiangsu Xing Textile Machinery Co., machine

Machine No.: E3/5/7 Variable pitch

Yarns: 28s/2、White, green, pink, red, blue, orange, mulberry /PP blended yarn

Systems: Zhejiang Heng Qiang technology co.ltd, heng qiang plate making software system.

\section{2 sample display}

The pipe part of the partial tubular flat knitting fabric composite preform has stereoscopic sense, Presents a tubular appearance, Multilayer composite sheets can be formed at one time. The fabrich have strong integrity. the prepared composite materials have superior performance. The sample of partial tubular flat knitting fabric composite preform made on transverse machine is shown in Figure 3. The composite sheet can be hot pressed into three layers at one time, and the state diagram of the fabric is shown in Figure 4.

Ltd. automatic computerized flat knitting

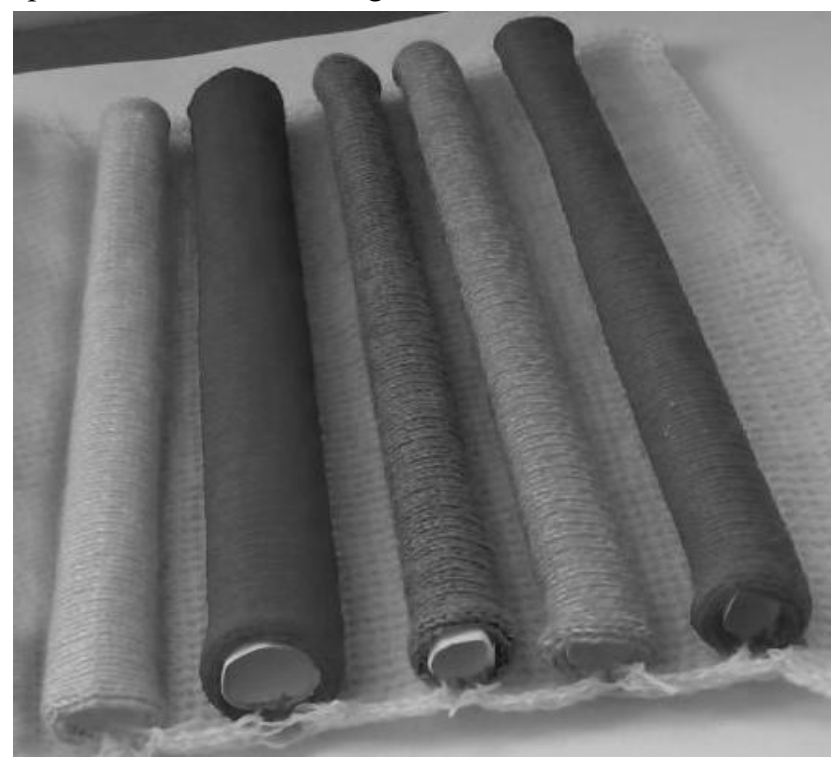

Figure 3 partial tubular flat knitting fabric composite preform

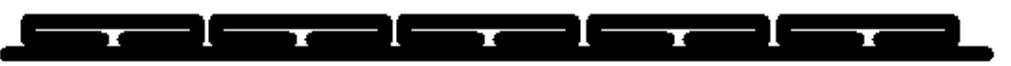

Figure 4 The state of partial tubular flat knitting fabric which was hot pressed into composite sheet

\section{5. revised design of partial tubular flat knitting fabric composite preform}

Braiding of partial tubular flat knitting fabric composite preform can be rivised by adjustingsome process parameters and the woven yarn.

\subsection{Change the row number of the pipe part}

During the development and design of the partial tubular flat knitting fabric composite preform, Change the row number of woven pipe part can change the size of the pipe part for example, increase the row number of yarn 2-6, The braided fabric's pipe part will become long. And the shape of the "tube" becomes larger. It will need lesser pipes for the same length of 
composite sheet. As shown in Figure 5.

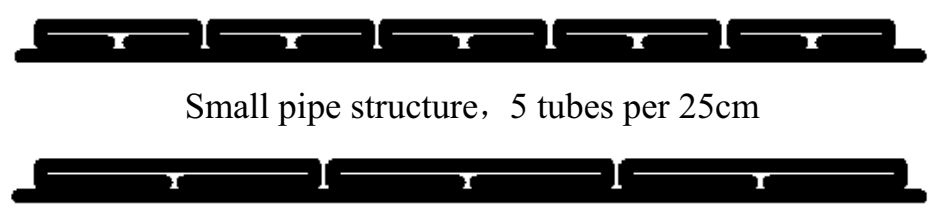

big pipe structure, 3 tubes per $25 \mathrm{~cm}$

Figure 5 Comparison of partial tubular flat knitting fabric with different pipes after hot pressing

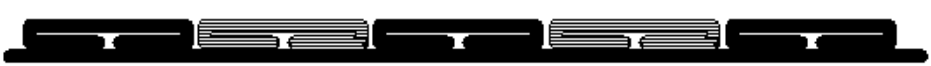

Weave the tubular part by using different raw materials 、 styles of yarns

Figure 6 change the tubular part yarn

5.2 Change the yarn in the pipe part Partial tubular flat knitting fabric has strong stereoscopic effect, and has the appearance of tubular structure. Using different yarn weaving, fabric surface presents different mutual spacing pipe which have different raw materials, different sizes, different styles of yarn. As shown in Figure 6, According to the different needs for different composite materials, we can choose the different yarns.

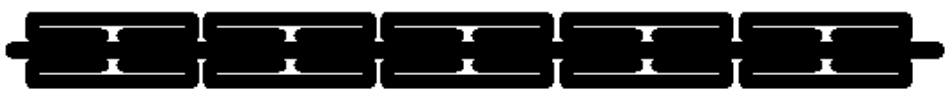

double-faced partial tubular flat knitting fabric's state after being hot-pressed

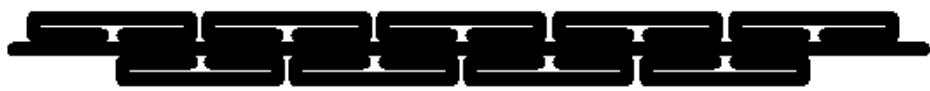

The state of the double side asymmetrical partial tubular flat knitting fabric after being hot pressed Fig. 7 contrast diagram of different double-faced partial tubular flat knitting fabric after being hot pressed

5.3 Design of double-faced partial tubular flat knitting fabric composite preform

when design the partial tubular flat knitting fabric composite preform, knit one side tubular fabric, forming Sheet of three layers fabric after hot pressing 。 knit two side tubular fabric, forming Sheet of five layers fabric after hot pressing。As shown in Figure 7,

when knit double-faced partial tubular flat knitting fabric composite preform, the double-faced pipe structure can be designed symmetrical or asymmetrical.

\section{Conclusions}

Through the structure design 、 weaving technology develop and test weaving of partial tubular flat knitting fabric composite preform,

The fabric's structure has strong stereoscopic sense, many kind of material and various style yarn can be chossed to knit, this [product has good market prospect and application value.

The partial tubular flat knitting fabric composite preform can be made into multilayer composite sheet one time, particular to the 5 layers, the composite materials have superior performance.

\section{References}

[1]TAO Li-jun,LIN Feng-ling,YIN Xiao-hai,LIU Xiao-hong,Preparation and Study on Weft Knitted Fabric Feinforced Composites [J].JOURNAL OF WUHAN UNIVERSITY OF SCIENCE AND ENGINERING,2010, 23 (4) : 4-7.

[2]Zhang Li,Ma Chongqi and Cheng Qingji, Research on Woven Method of a New Threedimensional Textile Composite Perform[J].INDUSTRIAL TEXTILE, 2006 (12) : 5-9.

[3] CHEN Jie,QIN Xiao,WANG Jianming, Development of partial tubular flat knitted fabrics[J].Journal of Textile Research， 2013,9 